\title{
Calculation of tunnel splitting in a biaxial spin particle with an applied magnetic field
}

\author{
Bin Zhou ${ }^{1,2,3}$, Shun-Qing Shen ${ }^{2}$, and Jiu-Qing Liang ${ }^{4}$ \\ ${ }^{1}$ Department of Physics, Fudan University, Shanghai 200433, China \\ ${ }^{2}$ Department of Physics, The University of Hong Kong, Hong Kong, China \\ ${ }^{3}$ Department of Physics, Hubei University, Wuhan 430062, China \\ ${ }^{4}$ Institute of Theoretical Physics, Shanxi University, Taiyuan 030006, China
}

(Dated: May 10, 2004)

\begin{abstract}
The level splitting formulae of excited states as well as ground state for a biaxial spin particle in the presence of an applied magnetic field are obtained in a simple way from Schrödinger theory. Considering the boundary condition of the wave function, we obtain the tunneling splitting of the energy levels for half-integral spins as well as for the integral spins. The results obtained are compared with those previously derived by complicated pseudoparticle methods and numerical calculation values.
\end{abstract}

PACS numbers: 75.45.+j Macroscopic quantum phenomena in magnetic systems - 75.50.Xx Molecular magnets - 73.40.Gk Tunneling 


\section{INTRODUCTION}

Quantum tunneling in spin systems has attracted considerable attention both theoretically and experimentally in view of a possible experimental test of tunneling effect for mesoscopic quantum tunneling $[1,2]$. In particular, the coherent tunneling between two degenerate metastable orientations of magnetization results in the superposition of macroscopically distinguishable states, i.e. macroscopic quantum coherence (MQC) [3]. Up to now, molecular magnets are the most promising candidates to observe MQC. An experimental observation of quantum phase interference in the presence of an external magnetic field along the hard axis for molecular magnet $\mathrm{Fe}_{8}$ has been reported [4]. On the other hand, a scheme to realize Grover's algorithms in molecular magnets was proposed [5, 6]. The calculation of tunneling splitting of the ground and the excited states of a magnetic particle is an interesting topic since the tunneling rate should be measured in resonance experiments [2]. The instanton method is a useful tool to study quantum tunneling effects and has been performed to derive the level splitting formulae with or without an applied magnetic field [1, 7-13]. However, the instanton path integral calculations are complicated, especially for the case of excited states the consideration of periodic or nonvacuum instantons implies more complicated calculations. A simple method developed is by means of high-order degenerate perturbation theory, which is first used by Garanin [14]. The perturbation approach has been extended to different models [15-19]. On the other hand, some authors also performed alternative simple way from Schrödinger theory with appropriate boundary conditions [2022 ], i.e., comparing the Schrödinger equation with that of a periodic potential for which the level splitting is possibly available. In Refs.[20, 21] tunnel splitting in a biaxial spin particle in the absence of an applied magnetic field was calculated in this way. The case with an applied magnetic field was also investigated by comparing the Schrödinger equation with the Mathieu equation [22]. In this paper, we improve the calcaulation of Ref.[22] and demonstrate that the results obtained in Refs. [7, 8] for spin tunneling between classically degenerate states in the presence of an applied magnetic field can be obtained in a very simple way by comparison of the Schrödinger equation with the Lamé equation for which the level splitting is available [23]. Considering the boundary condition of the wave function, we obtain the interesting tunneling splitting of the energy levels for half-integral spins as well as for the integral spins. Moreover the level splitting formulae of the excited states yield 
automatically, which are much more difficult to achieve with the instanton path integral method. Note that the tunnel splitting of the model considered here was calculated with perturbation approach in the linear order in the field by Garanin and Chudnovsky [17]. In the following we use a method by Ulyanov and Zaslavskii (UZ) [24], i.e., the potential field description of quantum spin systems, and begin with the Schrödinger equation of the spin particle.

\section{THE LEVEL SPLITTING}

We consider the biaxial ferromagnetic particle in an applied magnetic field which is described by the Hamilton operator

$$
\widehat{H}=K_{1} \widehat{S}_{z}^{2}+K_{2} \widehat{S}_{y}^{2}-H_{y} \widehat{S}_{y}
$$

with XOY easy plane and the easy $\mathrm{X}$-axis in the XOY-plane, the applied magnetic field $H_{y}$ along the medium axis direction, where $K_{1}, K_{2}$ with $K_{1}>K_{2}>0$ are the anisotropy constant, $\widehat{S}_{i}(i=x, y, z)$ are spin operators obeying the usual commutation relation $\left[\widehat{S}_{i}\right.$, $\left.\widehat{S}_{j}\right]=i \epsilon_{i j k} \widehat{S}_{k}$, (using natural units throughout, i.e. $\hbar=c=1$ ), $\epsilon_{i j k}$ is Levi-Civita symbol. Enz and Schilling used a different representation of the same biaxial anisotropy model that we investigate here $[7,8]$. The relation between anisotropy $A, B$ in Refs.[7, 8] (see Eq.(1) there) and ours, i.e., $K_{1}, K_{2}$, is seen to be $K_{1}=A+B, K_{2}=A$. In the present investigation we reexamine the quantum spin system in terms of a method developed by Ulyanov and Zaslavskii [24]. For convenience, the dimensionless parameters $k, \lambda$, and $\alpha$ are introduced as follows:

$$
k^{2}=\lambda=K_{2} / K_{1}, \alpha=H_{y} /\left(2 K_{2}(S+1 / 2)\right) .
$$

Following Ref.[24] we start from the Schrödinger equation

$$
\widehat{H} \Phi(\phi)=E \Phi(\phi)
$$

The explicit form of the action of the spin operator on the function $\Phi(\phi)$ is seen to be

$$
\widehat{S}_{z}=-i \frac{d}{d \phi}, \quad \widehat{S}_{x}=S \cos \phi-\sin \phi \frac{d}{d \phi}, \quad \widehat{S}_{y}=S \sin \phi+\cos \phi \frac{d}{d \phi},
$$

where the generating function $\Phi(\phi)$ is constructed in terms of the conventional spin functions 
of the $\widehat{S}_{z}$ representation such as

$$
\Phi(\phi)=\sum_{m=-S}^{S} \frac{c_{m}}{\sqrt{(S-m) !(S+m) !}} e^{i m \phi},
$$

which obviously obeys the following boundary condition

$$
\Phi(\phi+2 \pi)=e^{2 \pi i S} \Phi(\phi)
$$

Thus we have periodic wave functions for integer $S$ and antiperiodic functions for half-integer S. Substitution of the differential spin opertors Eq.(4) into Eq.(3) yields

$$
\begin{aligned}
0= & \left(K_{1}-K_{2} \sin ^{2} \phi\right) \frac{d^{2} \Phi}{d \phi^{2}}+\left[K_{2}\left(S-\frac{1}{2}\right) \sin 2 \phi-H_{y} \sin \phi\right] \frac{d \Phi}{d \phi} \\
& +\left(E-K_{2} S^{2} \cos ^{2} \phi-K_{2} S \sin ^{2} \phi+H_{y} S \cos \phi\right) \Phi
\end{aligned}
$$

where we have shifted the azimuthal angle by $\pi / 2$ for convenience $\phi+\pi / 2 \rightarrow \phi$. Then, we make use of the transformation [24-26],

$$
\Phi(\phi(x))=\operatorname{dn}^{S}(x) \exp [f(x)] \Psi(x)
$$

The new variable $x$ is defined by

$$
x=\int_{0}^{\phi} \frac{d \phi^{\prime}}{\sqrt{1-\lambda \sin ^{2} \phi^{\prime}}}=F(\phi, k),
$$

where $F(\phi, k)$ is the incomplete elliptic integral of the first kind with modulus $k^{2}=\lambda$, and $\operatorname{sn}(x), \operatorname{cn}(x)$, and $\operatorname{dn}(x)$ are the Jacobian elliptic functions with the same modulus. $f(x)$ is an auxiliary real function to be determined. Substituting Eq.(8) into (7), the eigenvalue equation is then transformed to the following effective potential form

$$
-K_{1} \frac{d^{2} \Psi}{d x^{2}}+V(x) \Psi=E \Psi
$$

where the scalar potential is

$$
V(x)=\left(K_{2} S(S+1)-\frac{H_{y}^{2}}{4 K_{1}}\right) \frac{\mathrm{cn}^{2}(x)}{\operatorname{dn}^{2}(x)}-H_{y}\left(S+\frac{1}{2}\right) \frac{\mathrm{cn}(x)}{\operatorname{dn}^{2}(x)}+\frac{H_{y}^{2}}{4 K_{1} \operatorname{dn}^{2}(x)} .
$$

The auxiliary function $f(x)$ is determined by elimination of the first derivative, such that

$$
\frac{d f(x)}{d x}=\frac{H_{y} \operatorname{sn}(x)}{2 K_{1} \operatorname{dn}(x)}
$$


From Eq.(10), we can formally write a point-particle like Hamiltonian as

$$
\hat{H}=\frac{\hat{P}^{2}}{2 m}+V(\hat{x})
$$

where $[\hat{P}, \hat{x}]=i$ and $m=1 /\left(2 K_{1}\right)$. The effective scalar potential $V(x)$ is a periodic function with period $4 K(k), K(k)$ denotes the complete elliptic integral of the first kind. The effective potential as a function of $x$ is plotted in Fig.1 where a constant has been added to make $V(x)$ zero at its minimum. From Fig.1, it is shown that for zero field case (i.e., $\alpha=0$ ) the effective potential $V(x)$ is a periodically symmetric double-well potential, while due to the applied magnetic field along the medium axis the effective potential becomes an asymmetric twin barriers potential including large barriers and small barriers. It is noted that in order to keep the shape of the asymmetric twin barriers potential, there exists a constraint condition (with $\left.S(S+1) \simeq(S+1 / 2)^{2}\right)$, i.e. $\alpha \lambda<(1-\lambda),(0<\lambda<1)$ and $0<\alpha<1$. In the new variables $x$ the wave function $\Phi[\phi(x)]$ is also periodic for integer $S$ and antiperiodic for half-integer $S$ with a period $4 K(k)$ and the boundary condition of the wave function $\Psi(x)$ is

$$
\Psi[x+4 K(k)]=e^{2 \pi i S} \Psi(x) .
$$

The boundary condition Eq.(14) plays an important role in the following calculation of the tunneling splitting. In the case of $H_{y}=0$, the eigenvalue equation can be transformed to the Lamé equation (the relation $\operatorname{sn}(x+3 K)=-\operatorname{cd}(x)$ has been used) [21]. The level splitting of the eigenvalue of the Lamé equation is not so well-known but available in Ref.[23] (also cited in Ref.[21]). In the following we discuss the case of $H_{y} \neq 0$.

The quantum states of the system (i.e., the degenerate states separated by infinitely high barriers) are determined by the oscillator approximation of the system around minimum positions $x_{0}$ of $V$ given by

$$
\operatorname{cn}\left(x_{0}\right)=\alpha, \quad \operatorname{sn}\left(x_{0}\right)= \pm \sqrt{1-\alpha^{2}}
$$

and so

$$
x_{0, n}^{+}=4 n K(k)+\mathrm{cn}^{-1} \alpha, \quad x_{0, n}^{-}=4 n K(k)-\mathrm{cn}^{-1} \alpha, \quad(n=0,1,2, \ldots),
$$

with

$$
\operatorname{sn}\left(x_{0, n}^{+}\right)=+\sqrt{1-\alpha^{2}}, \quad \operatorname{sn}\left(x_{0, n}^{-}\right)=-\sqrt{1-\alpha^{2}},
$$

at which

$$
V^{\prime \prime}\left(x_{0}\right)=2 K_{2} S(S+1)\left(1-\alpha^{2}\right) \equiv 8 h_{m}^{2} \cdot K_{1} .
$$


The Schrödinger equation $\hat{H} \Psi=E \Psi$ defined by $\hat{H}$ is then approximately

$$
\Psi^{\prime \prime}(x)+\left(\frac{E-V\left(x_{0}\right)}{K_{1}}-4 h_{m}^{2}\left(x-x_{0}\right)^{2}\right) \Psi \simeq 0 .
$$

This determines immediately the oscillator approximated eigenvalues as

$$
E_{2 n+1}^{(0)}=-\frac{H_{y}^{2}}{4 K_{2}}+(2 n+1) \sqrt{K_{1} K_{2} S(S+1)\left(1-\alpha^{2}\right)} .
$$

This perturbation theoretical expression ignores tunneling.

A typical aspect of any tunneling formula is the exponential of the Euclidean action of the classical vacuum pseudoparticle. This factor supplies the classical approximation of the transition amplitude equivalent to the wave function approximation given by the WKB exponential, so it vanishes in the limit of infinitely high barriers. In the present case this implies that $h_{m}^{2}$ (thus $S$ or $K_{2} S(S+1)$ ) has to be large. Therefore the argument of the exponential must contain $S$. In fact the factor in Eq.(9a) of Ref.[7] can be shown to approximate the WKB factor $\exp [-(S+1 / 2) \ln [(1+\sqrt{\lambda}) /(1-\sqrt{\lambda})]$ in the limit of vanishing magnetic field $H_{y}$. These observations suggest that the tunnel splitting is calculable for periodic potential by identification of appropriate parameters [22]. One avoids complicated integrals by setting in the Schrödinger equation $E=E_{2 n+1}^{(0)}+\Delta$, where $\Delta$ is the perturbation theory correction of the eigenvalue. The original Schrödinger equation then becomes

$$
\Psi^{\prime \prime}+\left(-G^{2}(x)+(2 n+1) \sqrt{\lambda S(S+1)\left(1-\alpha^{2}\right)}+\frac{\Delta}{K_{1}}\right) \Psi=0
$$

where

$$
G^{2}(x)=\lambda S(S+1)\left(\frac{\operatorname{cn}(x)-\alpha}{\operatorname{dn}(x)}\right)^{2} .
$$

Considering the boundary condition Eq.(14), we set

$$
\Psi=\Xi(x) \exp \left(-\int G(x) d x\right) \exp \left(\frac{2 \pi i S}{4 K} x\right),
$$

where $\Xi(x+4 K(k))=\Xi(x)$ and using the relations of $\operatorname{sn}(x+4 K)=\operatorname{sn}(x), \operatorname{cn}(x+4 K)=$ $\operatorname{cn}(x)$ and $\operatorname{dn}(x+4 K)=\operatorname{dn}(x)$. We obtain the WKB exponential

$$
\begin{aligned}
& \exp \left(-\int G(x) d x+\frac{2 \pi i S}{4 K} x\right) \\
= & \exp \left(-\sqrt{S(S+1)}\left[\ln \frac{1+\sqrt{\lambda} \operatorname{sn}(x)}{\operatorname{dn}(x)}-\alpha \sqrt{\frac{\lambda}{1-\lambda}} \tan ^{-1} \frac{\sqrt{1-\lambda} \operatorname{sn}(x)-\operatorname{cn}(x)}{\sqrt{1-\lambda} \operatorname{sn}(x)+\operatorname{cn}(x)}\right]\right) \\
& \times \exp \left(\frac{2 \pi i S}{4 K} x\right) .
\end{aligned}
$$


The boundary conditions require the evaluation of the wave function above from the chosen minimum of the potential (say the one at $x_{0,0}^{+}$), implying for the barries to the left and to the right

$$
\begin{aligned}
& \int_{x_{0,0}^{+}}^{x_{0,0}^{-}}\left(-G(x)+\frac{2 \pi i S}{4 K}\right) d x \\
= & \sqrt{S(S+1)}\left[\ln \frac{1+\sqrt{\lambda\left(1-\alpha^{2}\right)}}{1-\sqrt{\lambda\left(1-\alpha^{2}\right)}}-2 \alpha \sqrt{\frac{\lambda}{1-\lambda}} \tan ^{-1} \frac{\sqrt{(1-\lambda)\left(1-\alpha^{2}\right)}}{\alpha}\right] \\
& -\frac{i \pi S \mathrm{cn}^{-1} \alpha}{K}, \\
& \int_{x_{0,0}^{+}}^{x_{0,1}^{-}}\left(-G(x)+\frac{2 \pi i S}{4 K}\right) d x \\
= & \sqrt{S(S+1)}\left[\ln \frac{1+\sqrt{\lambda\left(1-\alpha^{2}\right)}}{1-\sqrt{\lambda\left(1-\alpha^{2}\right)}}+2 \alpha \sqrt{\frac{\lambda}{1-\lambda}} \tan ^{-1} \frac{\sqrt{(1-\lambda)\left(1-\alpha^{2}\right)}}{\alpha}\right] \\
& +2 \pi i S-\frac{i \pi S \operatorname{cn}^{-1} \alpha}{K} .
\end{aligned}
$$

The real parts of these expressions are seen to be (cf. Ref.[27]) precisely the values of the action of the instantons travelling through the two differently sized barriers between $\left(x_{0,0}^{+}\right.$, $\left.x_{0,0}^{-}\right)$and $\left(x_{0,0}^{+}, x_{0,1}^{-}\right)$respectively. It is exponential factors like those of Eq.(24) with the boundary conditions of Eqs.(25) and (26) which are typical tunneling contributions. In the present case both of these contribute to the overall level splitting. Knowing these factors we can write down the level splitting by making the appropriate replacements in the formula for the level splitting in the case of the Lamé equation (cf. Ref.[23], also cited in Ref.[21]) and adding these with equal weights so that in the limit $\alpha \rightarrow 0$ we regain the level splitting of the case without the magnetic field [22]. The factors multiplying the exponentials are characteristic of the central well (i.e., $h_{m}^{2}$ of Eq.(18)). Classically these factors describe the number of bounces of the particle between the barriers before it escapes. Thus these factors are the same in both cases so that from the level splitting result of Ref.[23] (also cited in Ref.[21]) the level splitting of the $n$th excited state in the present case is obtained by the 
replacement

$$
\begin{aligned}
& \exp \left[-\sqrt{S(S+1)} \ln \frac{1+\sqrt{\lambda}}{1-\sqrt{\lambda}}\right] \\
\longrightarrow & \frac{1}{2} \exp \left[-\sqrt{S(S+1)} \ln \frac{1+\sqrt{\lambda\left(1-\alpha^{2}\right)}}{1-\sqrt{\lambda\left(1-\alpha^{2}\right)}}\right] \\
& \times\left\{\exp \left[2 \alpha \sqrt{S(S+1)} \sqrt{\frac{\lambda}{1-\lambda}} \tan ^{-1} \frac{\sqrt{(1-\lambda)\left(1-\alpha^{2}\right)}}{\alpha}\right] \exp \left(\frac{i \pi S \mathrm{cn}^{-1} \alpha}{K}\right)\right. \\
& +\exp \left[-2 \alpha \sqrt{S(S+1)} \sqrt{\frac{\lambda}{1-\lambda}} \tan ^{-1} \frac{\sqrt{(1-\lambda)\left(1-\alpha^{2}\right)}}{\alpha}\right] \\
& \left.\times \exp \left(-2 \pi i S+\frac{i \pi S \mathrm{cn}^{-1} \alpha}{K}\right)\right\},
\end{aligned}
$$

and the level splitting in this general case becomes

$$
\begin{aligned}
\Delta_{2 n+1}= & \frac{4}{n !} 2^{3 n+2} \frac{\lambda^{(1 / 2)(n+1 / 2)}\left[S(S+1)\left(1-\alpha^{2}\right)\right]^{(1 / 2)(n+3 / 2)}}{(1-\lambda)^{n}}\left[\frac{K_{1} K_{2}}{(1-\lambda) \pi}\right]^{1 / 2} \\
& \times \exp \left[-\sqrt{S(S+1)} \ln \frac{1+\sqrt{\lambda\left(1-\alpha^{2}\right)}}{1-\sqrt{\lambda\left(1-\alpha^{2}\right)}}\right] \\
& \left|e^{i \pi S\left(\frac{\mathrm{cn}^{-1} \alpha}{K}-1\right)} \cos \left[S \pi-i 2 \alpha \sqrt{S(S+1)} \sqrt{\frac{\lambda}{1-\lambda}} \tan ^{-1} \frac{\sqrt{(1-\lambda)\left(1-\alpha^{2}\right)}}{\alpha}\right]\right| \\
& \times\left(1+O\left(\frac{1}{\kappa}\right)\right) .
\end{aligned}
$$

Here, $\kappa^{2}=\lambda S(S+1)$. We take the modulus of the phase factor not only because the energy must be positive, but also in agreement with the way in which the phase factor is handled in the path integral method (cf. Ref.[28]). For integral values of $S$ this formula reduces to

$$
\begin{aligned}
\Delta_{2 n+1}^{I}= & \frac{4}{n !} 2^{3 n+2} \frac{\lambda^{(1 / 2)(n+1 / 2)}\left[S(S+1)\left(1-\alpha^{2}\right)\right]^{(1 / 2)(n+3 / 2)}}{(1-\lambda)^{n}}\left[\frac{K_{1} K_{2}}{(1-\lambda) \pi}\right]^{1 / 2} \\
& \times \exp \left[-\sqrt{S(S+1)} \ln \frac{1+\sqrt{\lambda\left(1-\alpha^{2}\right)}}{1-\sqrt{\lambda\left(1-\alpha^{2}\right)}}\right] \\
& \cosh \left[2 \alpha \sqrt{S(S+1)} \sqrt{\frac{\lambda}{1-\lambda}} \tan ^{-1} \frac{\sqrt{(1-\lambda)\left(1-\alpha^{2}\right)}}{\alpha}\right]\left(1+O\left(\frac{1}{\kappa}\right)\right) .
\end{aligned}
$$

In the limit $\alpha \rightarrow 0$ this reduces to the formula obtained in Ref.[21] or to $\Delta E_{0}^{\text {inst }}$ of formula (9a) of Ref.[7]. For half-integral values of $S$ and applied field zero, i.e. $\alpha=0$, the splitting vanishes. The case is consistent with the Kramer's degeneracy. The interesting case is that 
of half-integral values of $S$ and magnetic field unequal zero. In this case we obtain

$$
\begin{aligned}
\Delta_{2 n+1}^{H}= & \frac{4}{n !} 2^{3 n+2} \frac{\lambda^{(1 / 2)(n+1 / 2)}\left[S(S+1)\left(1-\alpha^{2}\right)\right]^{(1 / 2)(n+3 / 2)}}{(1-\lambda)^{n}}\left[\frac{K_{1} K_{2}}{(1-\lambda) \pi}\right]^{1 / 2} \\
& \times \exp \left[-\sqrt{S(S+1)} \ln \frac{1+\sqrt{\lambda\left(1-\alpha^{2}\right)}}{1-\sqrt{\lambda\left(1-\alpha^{2}\right)}}\right] \\
& \sinh \left[2 \alpha \sqrt{S(S+1)} \sqrt{\frac{\lambda}{1-\lambda}} \tan ^{-1} \frac{\sqrt{(1-\lambda)\left(1-\alpha^{2}\right)}}{\alpha}\right]\left(1+O\left(\frac{1}{\kappa}\right)\right),
\end{aligned}
$$

which is a plausible result because it vanishes completely for vanishing magnetic field. In particular we obtain for $n=0$

$$
\begin{aligned}
\Delta_{1}^{I}= & 16\left[\frac{K_{1} K_{2}}{(1-\lambda) \pi}\right]^{1 / 2} \lambda^{1 / 4}\left[S(S+1)\left(1-\alpha^{2}\right)\right]^{3 / 4} \exp \left[-\sqrt{S(S+1)} \ln \frac{1+\sqrt{\lambda\left(1-\alpha^{2}\right)}}{1-\sqrt{\lambda\left(1-\alpha^{2}\right)}}\right] \\
& \cosh \left[2 \alpha \sqrt{S(S+1)} \sqrt{\frac{\lambda}{1-\lambda}} \tan ^{-1} \frac{\sqrt{(1-\lambda)\left(1-\alpha^{2}\right)}}{\alpha}\right]\left(1+O\left(\frac{1}{\kappa}\right)\right) \\
\Delta_{1}^{H}= & 16\left[\frac{K_{1} K_{2}}{(1-\lambda) \pi}\right]^{1 / 2} \lambda^{1 / 4}\left[S(S+1)\left(1-\alpha^{2}\right)\right]^{3 / 4} \exp \left[-\sqrt{S(S+1)} \ln \frac{1+\sqrt{\lambda\left(1-\alpha^{2}\right)}}{1-\sqrt{\lambda\left(1-\alpha^{2}\right)}}\right] \\
& \sinh \left[2 \alpha \sqrt{S(S+1)} \sqrt{\frac{\lambda}{1-\lambda}} \tan ^{-1} \frac{\sqrt{(1-\lambda)\left(1-\alpha^{2}\right)}}{\alpha}\right]\left(1+O\left(\frac{1}{\kappa}\right)\right) .
\end{aligned}
$$

The formula (31) for integral spins is to be compared with the corresponding path integral result of Ref.[8] (there Eq.(16)). In our result the origin of every factor is clearly understood as explained above. The somewhat different factors in Ref.[8] result from the complicated path integral calculation. The parameter $\kappa$ of Eqs.(28-32) has to be large, and the result is the dominant contribution of an asymptotic expansion in descending powers of $\kappa$. This condition implies that $S(S+1) \gg 1 / \lambda(0<\lambda<1)$. Since our calculations are done in the limit of large $S$, in prefactor one can replace $S(S+1)$ by $S^{2}$, and in the exponential factor $\sqrt{S(S+1)}$ has to be approximated by $S+1 / 2$.

In Fig. 2 we plot our expression $\Delta_{1}^{I}$ (in the limit of large $S$ ) for the values given in Fig.2 of Ref.[8]. In comparison with the results of Ref.[8], it is shown that for the small $\alpha$ values the plots of both results are fairly identical, but as $\alpha$ increases the plots our results deviate from those of Ref.[8]. In Tables 1 and 2 we display some absolute values of the level splitting as calculated from our result and compare these with values given in Ref.[7]. The relation between anisotropy $A, B$ in Refs.[7, 8] and ours, i.e., $K_{1}, K_{2}$, is $B=K_{1}-K_{2}, A=K_{2}$. 


\section{SUMMARY}

We investigate the biaxial spin system in the presence of an applied magnetic field with the potential field description of quantum spin system developed in Ref.[24]. Different from previous instanton technique or perturbation approach, we use an alternative simple way from Schrödinger theory. Considering the boundary condition of the wave function, we obtain the tunneling splitting of the energy levels for half-integral spins as well as for integral spins. The level splitting for half-integral spins vanishes completely for vanishing magnetic field due to Kramer's degeneracy. Moreover the level splitting formulae of the excited states yield automatically. Compared with the complicated instanton path integral method, the derivation of the nontrivial level splitting given here demonstrates the calculational simplicity of the Schrödinger method. Therefore, the method should appeal particularly to experimentists investigating macroscopic spin tunneling.

\section{Acknowlegements}

The work was supported by China Postdoctoral Science Foundation under Grant No. 2002032138, National Natural Science Foundation of China under Grant No. 10347106, and the Research Grants Council of Hong Kong under Grant No. HKU7023/03P.

[1] A. J. Leggett, S. Chakravarty, A. T. Dorsey, M. P. A. Fisher, A. Garg and W. Zwerger, Rev. Mod. Phys. 59, 1 (1987)

[2] Proceedings of the Meeting on Quantum Tunneling of Magnetization-QTM'94, edited by L. Gunther and B. Barbara, Vol. 301 of NATO ASI Series (Kluwer, Dordrecht, 1995)

[3] A. Garg, Phys. Rev. Lett. 71, 4249 (1993)

[4] W. Wernsderfer and R. Sessoli, Science 284,133 (1999)

[5] M. N. Leuenberger and D. Loss, Nature 410, 789 (2001)

[6] B. Zhou, R. Tao, S.-Q. Shen, and J.-Q. Liang, Phys. Rev. A 66, 010301 (2002)

[7] M. Enz and R. Schilling, J. Phys. C: Solid State Phys. 19, 1765 (1986)

[8] M. Enz and R. Schilling, J. Phys. C: Solid State Phys. 19, L711 (1986)

[9] E. M. Chudnovsky and L. Gunther, Phys. Rev. Lett. 60, 661 (1988) 
[10] D. Loss, D. P. DiVincenzo and G. Grinstein, Phys. Rev. Lett. 69, 3232 (1992)

[11] J.-Q. Liang, H. J. W. Müller-Kirsten, J.-G. Zhou and F.-C. Pu, Phys. Lett. A 228, 97 (1997)

[12] J.-Q. Liang, H. J. W. Müller-Kirsten, J.-G. Zhou and F. Zimmerschied, Phys. Lett. B 393, $368(1997)$

[13] J.-Q. Liang, Y.-B. Zhang, H. J. W. Müller-Kirsten, J.-G. Zhou, F. Zimmerschied and F.-C. Pu, Phys. Rev. B 57, 529 (1998)

[14] D. A. Garanin, J. Phys. A 24, L61 (1991)

[15] D. A. Garanin and E. M. Chudnovsky, Phys. Rev. B 56, 11102 (1997)

[16] D. A. Garanin and E. M. Chudnovsky, Phys. Rev. B 59, 3671 (1999)

[17] D. A. Garanin and E. M. Chudnovsky, Phys. Rev. B 65, 094423 (2002)

[18] M. N. Leuenberger and D. Loss, Phys. Rev. B 61, 1286 (2000)

[19] V. V. Ulyanov and O. B. Zaslavskii, Phys. Rev. B 60, 6212 (1999)

[20] J.-Q. Liang, H. J. W. Müller-Kirsten and J. M. S. Rana, Phys. Lett. A 231, 255 (1997)

[21] B. Zhou, J.-Q. Liang and F.-C. Pu, Phys. Lett. A 278, 95 (2000)

[22] J.-Q. Liang, H. J. W. Müller-Kirsten, A. V. Shurgaia and F. Zimmerschied, Phys. Lett. A 237, 169 (1998)

[23] H. J. W. Müller, Math. Nachr. 31, 89 (1966); 32, 49 (1966); 32, 157 (1966)

[24] V. V. Ulyanov and O. B. Zaslavskii, Phys. Rep. 216, 179 (1992)

[25] J.-Q. Liang, H. J. W. Müller-Kirsten, D. K. Park and F.-C. Pu, Phys. Rev. B 61, 8856 (2000)

[26] O. B. Zaslavskii, Phys. Lett. A 145, 471 (1990)

[27] Y.-H. Jin, Y.-H. Nie, J.-Q. Liang, Z.-D. Chen, W.-F. Xie and F.-C. Pu, Phys. Rev. B 62, 3316 (2000)

[28] H.-B. Braun and D. Loss, in Proceedings of the Meeting on Quantum Tunneling of Magnetization-QTM'94, edited by L. Gunther and B. Barbara, Vol. 301 of NATO ASI Series (Kluwer, Dordrecht, 1995), p. 319

\section{Figure captions}

Figure 1. The effective potential $V(x)$. The limit of large $S$ is considered. A constant has been added to make $V(x)$ zero at its minimum. The unit of $V(x)$ is $K_{2} S(S+1)$ and $\lambda=0.5$. The dotted line corresponds to $\alpha=0$; the solid line to $\alpha=0.2$; the dashed line to 
$\alpha=0.4$

Figure 2. Logarithmic plot of $\Delta_{1}^{I}$ (in units of $K_{2}$ ) as function of $\alpha$ for $\lambda=0.5$ and $S=5,10,20,30$ to be compared with Fig.2 of Ref.[8]. The solid lines correspond to our expression $\Delta_{1}^{I}$ (in the limit of large $S$ ), and the dotted lines to results of Ref.[8].

\section{Table captions}

\section{Table 1.}

Level splitting values calculated from $\Delta_{1}^{I}$ (in units of $K_{1}-K_{2}$ ) compared with the numerical values $\Delta E_{0}$ and the semiclassical results $\Delta E_{0}^{\text {inst }}$ of Ref.[7] for $S=10, K_{1}-K_{2}=1$ and different values of $K_{2}$ and $H_{y}$.

Table 2.

Level splitting values calculated from $\Delta_{1}^{H}$ (in units of $K_{1}-K_{2}$ ) compared with the numerical values $\Delta E_{0}$ for $S=10.5, K_{1}-K_{2}=1$ and different values of $K_{2}$ and $H_{y}$. 


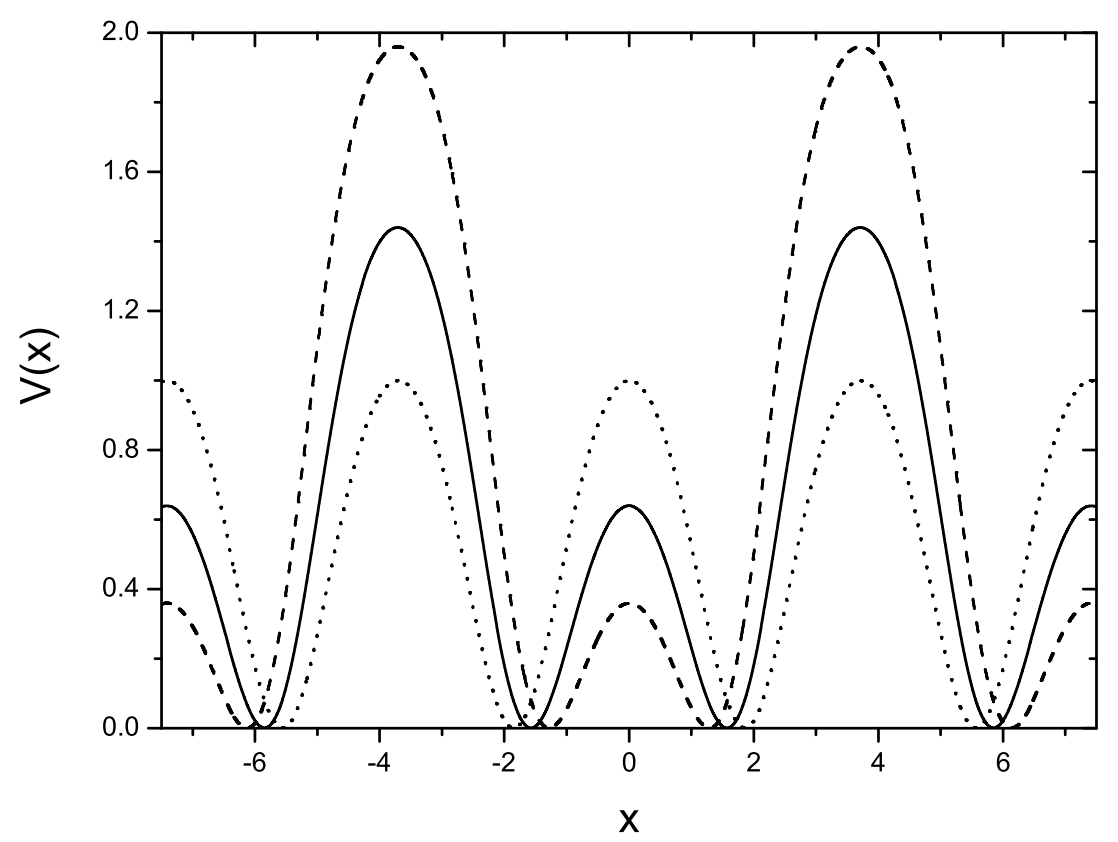

FIG. 1:

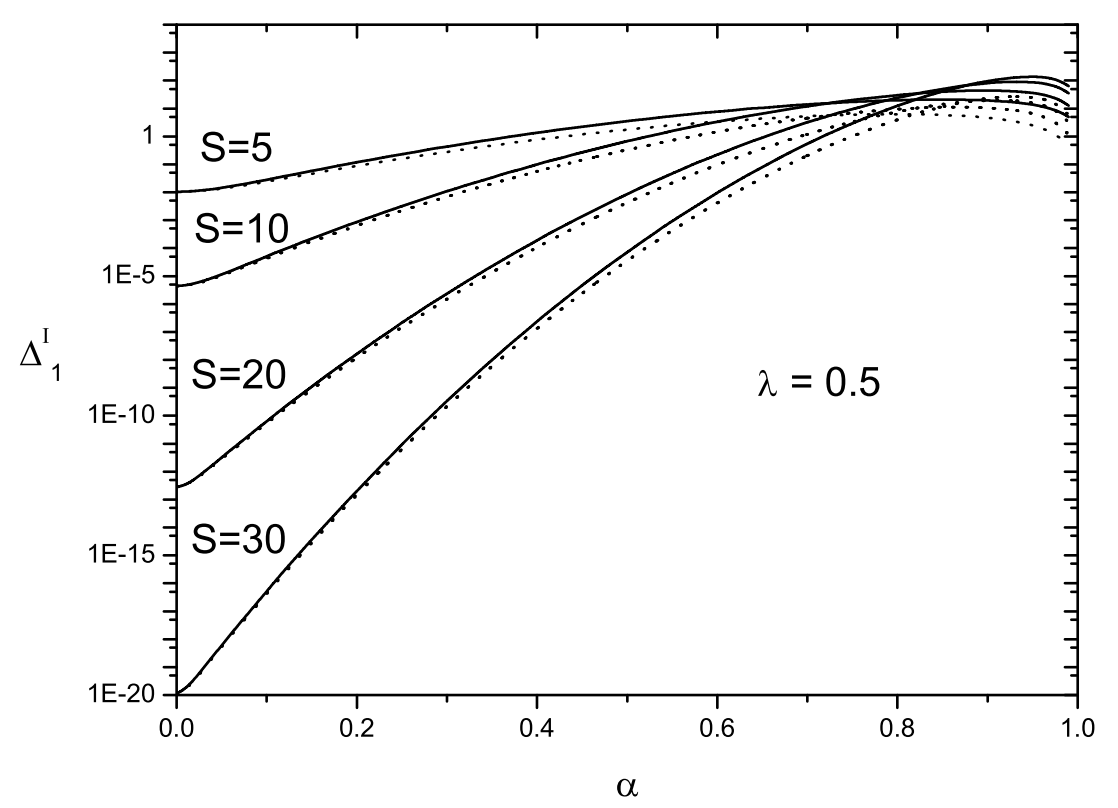

FIG. 2:

Table 1. 


\begin{tabular}{lllll}
\hline$K_{2}$ & $H_{y}$ & $\Delta_{1}^{I}$ & $\Delta_{1}^{I} / \Delta E_{0}$ & $\Delta E_{0}^{\text {inst }} / \Delta E_{0}$ \\
\hline 0.5 & 0 & $2.272 \times 10^{-4}$ & 1.029 & 1.029 \\
\hline & 0.4 & $3.209 \times 10^{-4}$ & 1.038 & 1.065 \\
\hline & 0.8 & $6.568 \times 10^{-4}$ & 1.043 & 1.123 \\
\hline & 1.2 & $1.447 \times 10^{-3}$ & 1.049 & 1.174 \\
\hline & 0 & $3.850 \times 10^{-8}$ & 1.015 & 1.015 \\
\hline & 0.4 & $4.238 \times 10^{-8}$ & 1.019 & 1.035 \\
\hline & 0.8 & $5.450 \times 10^{-8}$ & 1.021 & 1.083 \\
\hline & 1.2 & $7.684 \times 10^{-8}$ & 1.022 & 1.138 \\
\hline
\end{tabular}

Table 2.

\begin{tabular}{llll}
\hline$K_{2}$ & $H_{y}$ & $\Delta_{1}^{H}$ & $\Delta_{1}^{H} / \Delta E_{0}$ \\
\hline 0.5 & 0 & 0 & - \\
\hline & 0.4 & $1.248 \times 10^{-4}$ & 1.018 \\
\hline & 0.8 & $3.419 \times 10^{-4}$ & 1.027 \\
\hline & 1.2 & $7.988 \times 10^{-4}$ & 1.039 \\
\hline 2 & 0 & 0 & - \\
\hline & 0.4 & $5.992 \times 10^{-9}$ & 1.008 \\
\hline & 0.8 & $1.308 \times 10^{-8}$ & 1.007 \\
\hline & 1.2 & $2.261 \times 10^{-8}$ & 1.009 \\
\hline
\end{tabular}

